\title{
ASSOCIATION BETWEEN PERCEPTION OF ACCEPTANCE AND REJECTION, AND DRAWINGS OF "PERSON PICKING AN APPLE FROM A TREE" AMONG PRIMARY SCHOOL CHILDREN
}

\author{
Or Shalev ${ }^{1}$, Andriani Papadaki ${ }^{2}$, Elias Kourkoutas ${ }^{2}$, \& Michal Bat Or ${ }^{1}$ \\ ${ }^{\prime}$ The Graduate School of Creative Arts Therapies, University of Haifa (Israel) \\ ${ }^{2}$ Special Education and Psychology, University of Crete (Greece)
}

\begin{abstract}
The present study examined and compared associations between perceptions of parental acceptance/rejection in 644 Greek school age children (323 boys and 321 girls, age range 10-12), and their "Person Picking an Apple from a Tree" (PPAT) drawings. Perception of parental behavior was measured by the "Parental Acceptance-Rejection Questionnaire" (Rohner and Khaleque, 2005). Drawing content was analyzed quantitatively according to a reliable rating system called the Symbolic Content in PPAT drawings (SC-PPAT: Bat Or et al., 2014, 2017). We employed k-means cluster analysis and obtained three relatively discrete PPAT scripts. Drawing scripts were found to be associated with children's perceptions of parental behavior; these associations were found mainly among boys, especially to highly aggressive parents. These results demonstrate how empirical inquiry into PPAT content contributes to identifying implicit relational representations; furthermore, they reinforce the need to examine drawings from a holistic perspective, while focusing on the relational experience of children as expressed through their pictorial PPAT narratives.
\end{abstract}

Keywords: Parental acceptance-rejection, children, PPAT drawings, gender difference.

\section{Introduction}

The parent-child relationship is critical to child development, and considered to be the origins of later relational attributes and personal qualities in an adult (Clarke \& Scharff, 2014). This refers not only to "real" parent-child relationships, but also to internal mental representations of the relationships with others (Flanagan, 2016). The Interpersonal Acceptance-Rejection Theory (IPARTheory; Rohner, 2016) is an evidence-based theory pertaining to socialization and life span development of children and adults, initially focused mostly on the effects of perceived parental acceptance-rejection in childhood, and was formed mainly through the use of verbal tools such as interviews, and self-report questionnaires (Rohner, 2015). Parental acceptance-rejection refers to a bipolar dimension of parental warmth, with parental acceptance at the positive end of the continuum, and parental rejection at the negative end. Acceptance refers to parents' love, affection, care, comfort, support, or nurturance of children. Rejection refers to the absence or withdrawal of parental warmth, love, or affection from their children (Khaleque, 2015). According to Rohner $(1980,2004)$, children and adults organize their perceptions of parental acceptance-rejection around four universal categories: a) warmth/affection - the quality of the affectionate relationship between the parents and their children, and the physical, verbal, and symbolic behaviors parents use (or are perceived to use) to express these feelings and behaviors; b) hostility/aggression - either physical, verbal, active and/or passive, and problems with the management of hostility and aggression; c) indifference/neglect - a lack of parental concern or interest in the child; and d) undifferentiated rejection - the individuals' belief that his/ her parent/s do not really care about him or her, without necessarily being able to prove this based on their behavior. Two meta-analyses found that children who perceived themselves as accepted by their parents tended to have socially accepted behaviors and positive personality characteristics (Khaleque, 2013; Khaleque \& Rohner, 2012). At the same time, empirical studies worldwide have shown a correlation between parental rejection and children's psychological maladjustment; behavioral problems (including conduct disorder, externalizing behaviors, and delinquency); psychological disorders; and decreased school performance (Dwairy, 2010; Groh, Roisman, IJzendoorn, Bakermans-Kranenburg, \& Fearon, 2012; Miles \& Harold, 2003; Putnick et al., 2015). Some gender differences were found in children's perception of parental acceptance-rejection in recent years. In a sample of 168 Greek children (aged 7-12), both boys and girls tended to perceive their parents as accepting, but girls perceived significantly more maternal acceptance than boys did. 
There was significant correlation between psychological adjustment of boys and perceived paternal, but not maternal, acceptance (Giotsa \& Touloumakos, 2014).

Projective Drawings (PDs) have been proposed for testing and evaluating mental development and intelligence levels in children, and later expanded to provide additional information about the individual, such as personality traits and emotional and cognitive development. A large body of evidence suggests that there are differences between the drawings of boys and those of girls, which can manifest in the pictorial content (Turgeon, 2008; Wright \& Black, 2013). The "Person Picking an Apple from a Tree" (PPAT) drawing task (Gantt, 1990) is an art-therapy assessment that has been found to be valuable in revealing associations between emotional-behavioral problems and cognitive (executive functions) aspects in preschool children (Bat Or, Ishai, \& Levi, 2014) and highly aggressive school-age children (Bat Or, Kourkoutas, Smyrnaky, \& Potchebutzky, in press). Significant associations between PPAT drawings and relationship representations has only been found among adults (Bat Or \& Ishai, 2016), whereas there is still a need to study these possible associations among children. The present study examined whether the content of children's PPAT drawing task (Gantt, 1990) was associated with their perceived parental attitude (acceptance and rejection) as measured by the "Parental Acceptance-Rejection Questionnaire" (Child PARQ; Rohner \& Khaleque, 2005).

Our research hypotheses were: (a) Associations will be found between perceived parental acceptance-rejection and the pictorial contents of PPAT drawings. Specifically, parental acceptance will correlate with the positive aspects of PPAT drawings (for example, active drawn person, successful picking, tree with apples); and, parental rejection will correlate with negative aspects in the PPAT (for instance, weak tree, passive drawn person, tree inclining away from the person, etc.). (b) Associations between PARQ and PPAT pictorial content will differ substantially between genders.

\section{Method}

\subsection{Participants}

The sample of 644 Greek school age children (age range 10-12) were randomly selected from public schools in three prefectures of the island of Crete (Heraklion, Chania, and Rethymnon). 277 children were from the fifth grade, and 367 from the sixth grade, $51 \%$ of the participants were boys and $49 \%$ were girls. Participant distribution was $86 \%$ urban residents and $14 \%$ semi urban residents.

\subsection{Instruments}

2.2.1. Parental Acceptance-Rejection Questionnaire (Child PARQ). Parental Acceptance-Rejection Questionnaire (Child PARQ) (Rohner, 1990; Adaptation in Greek in Giovazolias et al., 2010). The current study used the short form of the Parental Acceptance-Rejection Questionnaire: Child version (Child PARQ: Mother version, Child PARQ: Father version; Rohner and Khaleque, 2005). The Child PARQ short version encompasses 24 items and asks children to interpret their caregiver's behavior through their own personal experiences. Participants were asked to evaluate each statement on a four-point Likert scale ranging from 1 (almost never true) to 4 (almost always true). The scales were summed and keyed in the direction of perceived rejection. Mother and Father Child PARQ questionnaires are identical. The Warmth/Affection Scale is composed of eight items, for example, "My father/mother says nice things about me." Scores were inverted, thus high scores indicate lack of parental Warmth/Affection. The Hostility/Aggression Scale is composed of six statements, for example, "My father/mother hits me, even when I do not deserve it." The Indifference/Neglect Scale has six items, including statements such as "My father/mother pays no attention to me." Finally, the Undifferentiated Rejection Scale incorporates four statements such as "My father/mother seems to dislike me." The Greek Child PARQ was found to be a reliable and valid instrument (Artemis and Touloumakos, 2016). In the current study, the internal consistency of the total PARQ scores of mothers and fathers in each sub-scale were good (Cronbach's alphas were 0.853 and 0.851 , respectively, $\mathrm{N}=644$ ).

2.2.2. "Person Picking an Apple from a Tree" Drawing Task (PPAT). "Person Picking an Apple from a Tree" drawing task (Gantt, 1990, Unpublished). We followed the instructions proposed by Gantt and Tabone (1998) for administration of the PPAT process. Accordingly, participants were given white sheets of paper (21by $29.5 \mathrm{~cm}$ ) and markers in 12 colors (red, orange, blue, turquoise, green, dark green, hot pink, gray, purple, brown, yellow, and black), and were asked to draw "a person picking an apple from a tree" (Gantt and Tabone, 1998). Due to the slightly different composition of colors in the 12 pack markers sold in Greece, the gray colored marker replaced the magenta color noted in the original Gantt and Tabone (1998) instructions. The 'Symbolic Contents in "Person Picking an Apple from a Tree" for school-age children' (SC-PPAT/c2 Bat Or et al., 2017), comprises nine Likert-scales that range between 0 (the rated feature is absent) and 5 or 6 (the rated feature at its maximum). The scales measure three central aspects of the PPAT drawing: characteristics of the tree (for example the number of apples on the tree); characteristics of the person (for instance, the degree in which a person is active/passive in 
the apple picking process); and characteristics of the tree-person relationship (for example, the position of the tree truck in relation to the person's location). The drawings $(\mathrm{N}=644)$ were rated according to the SC-PPAT/c2 rating system; two trained raters coded $10 \%$ of each of the drawings, until they achieved substantial agreement. The inter-rater reliabilities were calculated by the Intra-Class Correlation coefficient, which ranged between good and excellent.

\subsection{Procedure}

Researchers initially secured approval from the Educational Institute of the Ministry of Education as well as the ethics committee of the University of Crete. Furthermore, meetings were held with the parents of the participants to inform them of the purposes of this research. Parents were asked to sign consent forms. The research was conducted in the schools, and researchers entered the class accompanied by the class teacher. On the first day, the researchers introduced themselves and administered the Child PARQ-mother/father questionnaires, and on the second day they administered the PPAT drawing task. Participants were individually asked to draw a person picking an apple from a tree; no time limitation was set. Researchers assured the children that there were no right or wrong answers, and no drawing would be considered an ugly drawing. They informed the children that the questionnaires and the drawings would be collected by the researchers.

\section{Results}

\subsection{SC-PPAT Factor Analysis and Cluster Analysis}

Three main factors were obtained by Confirmatory Factor Analysis. Each factor consists of two scales. Person Agency pertains to the drawn person's activity, including the degree in which the person is active/passive in the apple picking, and if there is contact between person and tree. Tree Accessibility pertains to the tree's orientation toward the drawn person, including the position of the tree trunk in relation to the person, and placement of branches in relation to the person. Finally, Tree Potency pertains to the characteristics of the tree, including the number of apples it bears and its strength vs. weakness. These factors yield a total of $68 \%$ of the explained variance. Inter-factor associations were also measured. The main finding is a medium positive association between Person Agency and the Tree Accessibility. In specific, as much as the person is more agent, the tree is more accessible for the person. K-means cluster analysis was conducted for identifying groups of PPAT drawings with different combinations of PPAT's main factors values. One-way ANOVA showed significant differences between the three main factors in each cluster. As can be seen in Table 1, three clusters were identified in PPAT drawings. Figure 1 illustrates drawings in cluster A: a potent tree, a low agent person, and a neutral-to-less-accessible tree $(n=295)$; Figure 2 illustrates drawings in cluster B: a non-potent tree, a person with medium agency, and a fairly accessible tree $(n=153)$; Finally, Figure 3 illustrates PPAT drawings in cluster C: a potent tree, a person with agency, and an accessible tree $(n=307)$. In terms of the PPAT narrative, cluster $\mathrm{C}$ describes the best scrip, in terms of coherency and reciprocity, while the two other clusters reveal gaps between personal agency and tree potency. While cluster B describes a narrative with a weak though accessible tree and medium personal agency, cluster A reflects a potent though less accessible tree and low personal agency. For the whole sample, children who drew cluster B PPAT tended to perceive their mothers as more rejecting, in comparison to children who drew cluster C PPAT.

Table 1. Final Cluster Centers.

\begin{tabular}{lccc}
\hline & & Cluster & \\
\cline { 2 - 4 } PPAT's main factors & $\mathrm{A}$ & $\mathrm{B}$ & $\mathrm{C}$ \\
\hline Potency of tree & 4.80 & 2.77 & 4.87 \\
Agency of person & 2.06 & 2.75 & 3.35 \\
Accessibility of tree & 2.69 & 2.86 & 2.99 \\
Number of PPAT & 295 & 153 & 307 \\
\hline
\end{tabular}

3.1.1. Gender differences. Pearson's correlation-coefficients were calculated between PARQ categories and SC-PPAT/c2 factors for both boys and girls. Person Agency was the only main factor associated with PARQ categories. Person Agency was negatively associated with the mother's Hostility/Aggression, $\mathrm{r}=-.207, \mathrm{p}<0.01$; in other words, the more the boy perceived his mother as hostile and aggressive towards him, his drawn person's agency was lower, and vice versa: the less hostile and aggressive the boy perceived his mother to be, the more agent person was drawn. In order to examine the differences in correlations that were found between the boys' group and the girls' group, z-tests for differences between Pearson's correlations - coefficients were conducted. Negative significant differences between boys and girls were found, correlated in the boys group $(r=-.207, \mathrm{p}<.001)$ but not in the girls 
group $(\mathrm{r}=-.017, \mathrm{~ns}), \mathrm{z}=2.33, \mathrm{p}<0.05$. In addition to these analyses, a one-way ANOVA analysis was conducted in each gender group between the three clusters and validity variables. Among boys, two significant differences relating to parental hostility levels were found. Specifically, a significant difference between clusters $\mathrm{A}$ and $\mathrm{C}$ in terms of their scores of father's hostility $\mathrm{F}(2,347)=3.42$, $\mathrm{p}=.034$; and a significant difference between clusters $\mathrm{A}$ and $\mathrm{C}$ in terms of their scores of mother's hostility $\mathrm{F}(2,337)=3.72, \mathrm{p}=.025$ were detected. In other words, only among boys was a significant difference found between cluster A and C (between PPAT with a potent tree, a low agent person, and a neutral to less-accessible tree and PPAT with coherent and reciprocal relations between the person and the tree. i.e., they are both potent and the tree is also accessible) in relation to reports about parental hostility levels. By the same token, boys who reported higher parental hostility tended to draw cluster A PPAT (with a mixed script: a potent tree, a person with low agency, and a neutral to a less-accessible tree) in comparison to boys that reported lower parental hostility levels and tended to draw cluster C PPAT (with a coherent script, in which all the three factors -a potent and accessible tree, and a person with agency_are positive).

\section{Figure 1. Cluster A PPAT with a potent and neutral tree in terms of accessibility, and a person with low agency.}

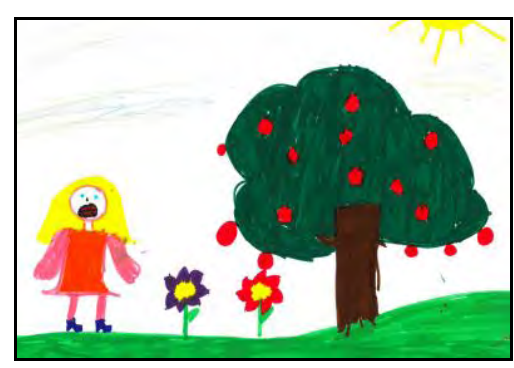

Figure 2. Cluster B PPAT with a non-potent and accessible tree, and a person with medium agency.

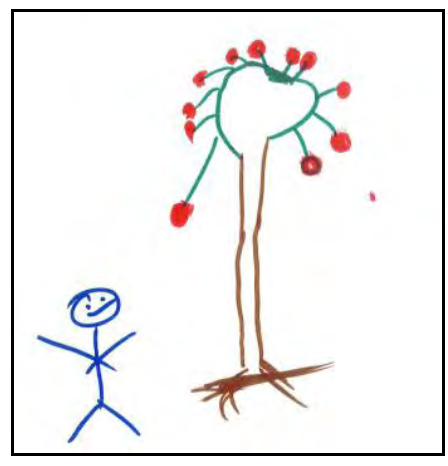

Figure 3. Cluster C PPAT with a potent and accessible tree, and $a$ person with agency.

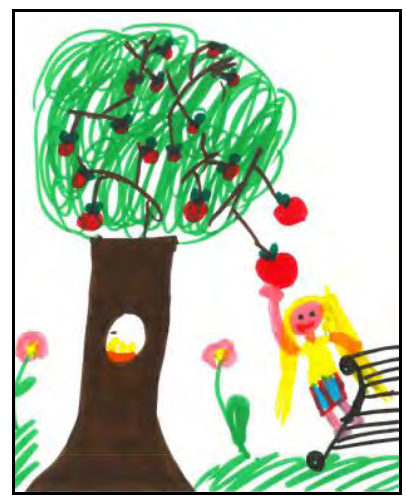

\section{Discussion and conclusion}

The present study contributed to the validity of the SC-PPAT rating system among school age children with a self-report relational measure (perception of parental acceptance-rejection). The preliminary analysis showed that the PPAT drawings of school age children, like those as adults comprise three main content factors: the tree potency, the tree accessibility, and the drawn person's agency. This supports the claim about the efficiency of the holistic approach in analysing projective drawings.(McGrath \& Carroll, 1992). Results demonstrated that certain PPAT narratives were associated with maternal hostility: specifically, children that reported higher levels of maternal rejection tended to draw a less potent tree and a person with neutral to low agency. These drawn objects might be representative of the children's internal working models, and thus influenced by their relational expectations, i.e. their hope to receive assistance from other people, how cooperatively they interact, their self-worth, and their ability to achieve goals (Grossmann, Grossmann, \& Waters, 2006). As regards to gender diferences, associations between parental hostility and a non-coherent PPAT script were found only among boys. The results indicate that the pictorial space of children's drawings, communicated through various symbolization and a thematic script may reveal their subjective experience (Malchiodi, 1998). This research establishes that a broader observation of drawing narratives or script is required to understand the child's subjective relational experience, especially when observing normative middle-childhood children, as was the case in this study. This is similar to clinical work with clients, where clinicians attempt to gain access to the client's relational scripts through personal narratives (McLeod, 1997).

\section{References}

Artemis, G., \& Touloumakos, A. K. (2016). "They Accept Me, They Accept Me Not” Psychometric Properties of the Greek Version of the Child Parental Acceptance Rejection Questionnaire-Short Form. Journal of Family Issues, 37(9), 1226-1243.

Bat Or, M., \& Ishai, R. (2016). Form and content in adults 'Person Picking an Apple from a Tree' drawings as related to their attachment. International Journal of Art Therapy, 21(1), 2-14. 
Bat Or, M., Ishai, R., \& Levi, N. (2014). Validating PPAT's symbolic meanings of emotional and cognitive functioning among children. The Arts in Psychotherapy, 41(4), 309-319.

Bat Or, M., Ishai, R., \& Levi, N. (2017). Symbolic Contents in PPAT drawings (SC-PPAT/c): Children (10-12 years). A Manual for rating PPAT drawings. University of Haifa.

Clarke, G. S., \& Scharff, D. E. (2014). Fairbairn and the object relations tradition. London: Karnac Books.

Dwairy, M. (2010). Parental acceptance-rejection: a fourth cross-cultural research on parenting and psychological adjustment of children. Journal of Child and Family Studies, 19(1), 30-35.

Flanagan, L. M. (2016). Object relations theory. In J. Berzoff, L. M. Flanagan, P. Hertz (Eds). Inside out and outside in: Psychodynamic clinical theory and psychopathology in contemporary multicultural contexts $\left(4^{\text {th }}\right.$ ed, pp. 123-165). Rowman \& Littlefield.

Gantt, L. (1990). A validity study of the Formal Elements Art Therapy Scale (FEATS) for diagnostic information in patients' drawings (Unpublished doctoral dissertation). University of Pittsburgh, Pittsburgh, PA.

Gantt, L. (2004). The Case for Formal Art Therapy Assessments. Art Therapy: Journal of the American Art Therapy Association, 21(1), 18-29.

Gantt, L., \& Tabone, C. (1998). The Formal Elements Art Therapy Scale: A rating manual. Morgantown, WV: Gargoyle Press.

Giotsa, A., \& Touloumakos, A. K. (2014). Perceived Parental Acceptance and Psychological Adjustment The Moderating Role of Parental Power and Prestige Among Greek Pre-Adolescents. Cross-Cultural Research, 48(3), 250-258.

Giovazolias, T., Kothali, P., Louvrou, B., \& Mitsopoulou, E. (2010). The Role of Father Involvement in Children's Bullying Behavior: Empirical Findings from a Greek Sample. 3rd Congress on Interpersonal Acceptance-Rejection. Padua, Italy: 28-31 July.

Groh, A.M., Roisman, G.I., van IJzendoorn, M.H., Bakermans-Kranenburg, M.J., \& Fearon, R.P. (2012). The significance of insecure and disorganized attachment for children's internalizing symptoms: A meta-analytic study. Child Development, 83, 591-610.

Grossmann, K. E., Grossmann, K., \& Waters, E. (2006). Attachment from infancy to adulthood: The major longitudinal studies. New York, NY: Guilford Press.

Khaleque, A. (2013). Perceived parental warmth, and children's psychological adjustment, and personality dispositions: A meta-analysis. Journal of child and Family studies, 22(2), 297-306.

Khaleque, A. (2015). Parental acceptance and children's psychological adjustment. In Promoting Psychological Well-Being in Children and Families (pp. 226-243). UK: Palgrave Macmillan.

Khaleque, A., \& Rohner, R. P. (2012). Pancultural associations between perceived parental acceptance and psychological adjustment of children and adults: A meta-analytic review of worldwide research. Journal of cross-cultural Psychology, 43(5), 784-800.

Malchiodi, C. (1998). Understanding children's drawings. New York: Guilford Press.

McLeod, J. (1997). Narrative and psychotherapy. London: Sage.

Miles, C. J., \& Harold, G. T. (2003). Parents warmth and hostility, children's depression and aggression: A cross-lagged, longitudinal analysis. Paper presented at the meeting of the Society for Research in Child Development, Tampa, FL.

Putnick, D. L., Bornstein, M. H., Lansford, J. E., Malone, P. S., Pastorelli, C., Skinner, A. T., \& Alampay, L. P. (2015). Perceived mother and father acceptance-rejection predict four unique aspects of child adjustment across nine countries. Journal of Child Psychology and Psychiatry, 56(8), 923-932.

Rohner, R. P. (1980). Worldwide tests of parental acceptance-rejection theory: An overview. Cross-Cultural Research, 15(1), 1-21.

Rohner, R. P. (1990). Handbook for the study of parental acceptance and rejection ( $3^{\text {rd }}$ ed.). Storrs, CT: Rohner Research Publications

Rohner, R. P. (2015). Itroduction to interpersonal acceptance-rejection theory (IPARTheory), methods, evidence, and implications. Retrieved from http://scholarworks.gvsu.edu/orpc/vol6/iss1/4/

Rohner, R. P. (2016). Introduction to Interpersonal Acceptance-Rejection Theory (IPARTheory) and Evidence. Online Readings in Psychology and Culture, 6(1), 4.

Rohner, R. P., \& Khaleque, A. (2005). Parental acceptance-rejection questionnaire (PARQ): Test manual. Handbook for the study of parental acceptance and rejection, 4, 43-106.

Teglasi, H. (2013). The Scientific Status of Projective Techniques as Performance Measures. In D. H. Saklofske, C. R. Reynolds, \& V. L. Schwean (Eds.). The Oxford handbook of child psychological assessment. (pp. 113-128). New York: Oxford University Press.

Wright, L., \& Black, F. (2013). Monochrome males and colorful females: do gender and age influence the color and content of drawings?. SAGE Open (4)1-9. 